\title{
Clinical Availability of ATRA for Patients With Suspected Acute Promyelocytic Leukemia: Why Guidelines May Not Be Followed
}

\author{
Marcus J. Geer, MD'; Charles E. Foucar, MD'; Sumana Devata, MD²; Lydia Benitez, PharmD³; \\ Anthony J. Perissinotti, PharmD ${ }^{3}$; Bernard L. Marini, PharmD ${ }^{3}$; and Dale Bixby, MD, PhD ${ }^{1}$
}

\begin{abstract}
Background: All-trans retinoic acid (ATRA) serves as the backbone of the management of patients with acute promyelocytic leukemia (APL), with guidelines recommending the initiation of ATRA as soon as APL is suspected. As a regional referral center for patients with acute leukemia, those who are suspected of having APL are often transferred to our facility. However, many referring centers are unable to initiate treatment using ATRA. We conducted an exploratory analysis of the clinical availability of ATRA and the factors limiting access to this critical drug. Patients and Methods: The United States was divided into 6 geographic regions: Northwest, Southwest, Central, Southeast, Northeast, and the Great Lakes. Twenty hospitals were randomly selected from states within each of these regions and were surveyed as to whether they typically treated patients with acute leukemia, the availability of ATRA at their institution, and reported reasons for not stocking ATRA (if not available). Results: Less than one-third of hospitals queried (31\%) had ATRA in stock. Neither the size of the hospital nor the hospital's status as academic versus nonacademic $(53 \%$ vs $31 \% ; P=.08$ ) influenced ATRA availability. Of the hospitals that referred patients with APL, only $14 \%$ (7/49) had ATRA readily available. Hospitals that treated patients with APL were more likely to have ATRA available than referring centers ( $58 \%$ vs $14 \% ; P=.000002$ ). Conclusions: Nearly two-thirds of the hospitals surveyed that cared for patients with acute leukemia do not have ATRA immediately available. Moreover, the vast majority of hospitals that refer patients to other centers do not have ATRA. These findings should spur investigation into the impact of immediate ATRA availability on the morbidity and mortality of patients with APL. A call by hematologists nationwide to their formulary committees is warranted to ensure that this lifesaving medication is available to patients suspected of having APL.
\end{abstract}

J Natl Compr Canc Netw 2021;19(11):1272-1275 doi: $10.6004 /$ jnccn.2021.7013

'Division of Hematology and Medical Oncology, Department of Internal Medicine, Michigan Medicine and University of Michigan Medical School, Ann Arbor, Michigan; ${ }^{2}$ Division of Hematology and Medical Oncology, Department of Internal Medicine, Medical College of Wisconsin, Milwaukee, Wisconsin; and ${ }^{3}$ Department of Pharmacy Services and Clinical Science, Michigan Medicine, and University of Michigan College of Pharmacy, Ann Arbor, Michigan.

\section{Background}

Acute promyelocytic leukemia (APL) is recognized as a true hematologic emergency, and all-trans retinoic acid (ATRA; tretinoin, Teva Pharmaceutical Industries Ltd) serves as a uniform backbone of the early care and management of patients with APL. ${ }^{1}$ SEER-17 data from 2001 to 2007 showed that APL represented $7.4 \%$ of all patients diagnosed with acute myeloid leukemia (AML), with an incidence rate of $2.7 \% .^{2}$ Although the 5 -year survival is higher in APL than in other AML subtypes, the 30-day mortality for APL is noteworthy, ranging from $15 \%$ to $25 \%{ }^{3}$ Proposed reasons for early mortality in APL include disease-related complications such as disseminated intravascular coagulopathy (DIC; bleeding, intracranial hemorrhage), treatment-related complications, and a lack of timely initiation of treatment. ${ }^{2,4}$ Although ATRA was first investigated as a salvage therapy for patients with relapsed or refractory disease, current European LeukemiaNet guidelines $^{5}$ and NCCN Clinical Practice Guidelines in Oncology (NCCN Guidelines) for $\mathrm{AML}^{6}$ call for the early use of ATRA in patients suspected of having APL, even before genetic confirmation of the disease. Furthermore, because ATRA can significantly mitigate DIC, known to be one of the early complications of APL, the NCCN and European LeukemiaNet guidelines support the prescription of ATRA as soon as there is clinical suspicion of the diagnosis. ${ }^{5,6}$

As a regional referral center for the care of patients with advanced myeloid malignancies, our facility receives numerous requests for the transfer of care of patients suspected of having APL. Consider the case of a female patient aged 63 years who initially presented to her primary care provider after experiencing a week of fatigue and easy bruising. She was referred to the nearest community emergency room for further evaluation when initial laboratory work revealed a new pancytopenia accompanied by profound thrombocytopenia. Upon hematopathologist review of a bone marrow aspirate, she was found to have features consistent with APL. The patient was given appropriate supportive care with transfusion of blood products, and our facility was contacted with a request for transfer. 
Given the concern for APL, immediate initiation of ATRA was recommended before transfer. Unfortunately, due to limited bed availability, transfer was delayed several days. In the interim, her condition continued to deteriorate, requiring ICU admission for DIC and thus dramatically increasing the risk of poor outcomes during transfer. Upon the patient's eventual transfer, a review of records revealed that ATRA had never been started.

Unfortunately, the circumstances surrounding this patient are not unique. The primary outcome of this study was to determine the clinical availability of ATRA for patients with a suspected diagnosis of APL at regional medical centers throughout the United States. The secondary objective was to identify the potential hurdles limiting the availability of ATRA at these institutions.

\section{Patients and Methods}

The United States was divided into 6 geographic regions: Northwest, Southwest, Central, Southeast, Northeast, and the Great Lakes. A state from each of these regions was randomly selected (Washington, Arizona, Missouri, Georgia, Massachusetts, and Michigan). Hospitals with a capacity of $>100$ beds were chosen from the American Hospital Directory (http:/ /www.ahd.com/), and all hospitals were assigned a number. These numbers were entered into a random number generator, and the first 20 hospitals from each region (120 total) were selected for the survey (excluding repeats). Two hospitals were excluded because of a lack of response after repeated attempts. The following questions were asked of the hospital's inpatient pharmacist:

1. Does your hospital treat acute leukemia or does it refer to other hospitals?

2. Do you have ATRA (oral), $10 \mathrm{mg}$ tablets on the formulary or immediately available in stock as a nonformulary request?

3. If no, why not?

Statistical analyses were performed using SPSS Statistics, version 20.0 (IBM Corp.). Quantitative variables are reported as the number (percentage) and dichotomous variables were compared using the chi-square test. A value of $P<.05$ was considered significant.

\section{Results}

ATRA was available in less than one-third of the hospitals queried (31\%; Table 1). There were no identifiable differences in the availability of ATRA based on hospital size (inpatient beds) or academic versus nonacademic status of the hospital (53\% vs $35 \% ; P=.08$ ). In hospitals that referred to other institutions for the care of their patients with leukemia $(49 / 118 ; 41.5 \%)$, only $14 \%(n=7)$ had
Table 1. Immediate Availability of ATRA by Region

\begin{tabular}{|lcc|}
\hline Region & State & Hospitals With ATRA (\%) \\
\hline Northwest & Washington & $13 / 20(65)$ \\
\hline Southwest & Arizona & $9 / 20(45)$ \\
\hline Central & Missouri & $4 / 20(20)$ \\
\hline Great Lakes & Michigan & $11 / 19(58)$ \\
\hline Southeast & Georgia & $7 / 20(35)$ \\
\hline Northeast & Massachusetts & $4 / 19(21)$ \\
\hline All regions & All states & $37 / 118(31)$ \\
\hline
\end{tabular}

Abbreviation: ATRA, all-trans retinoic acid.

ATRA on their formulary or available in stock as a nonformulary request that could act as a bridge before patient transfer. There was a significant difference in hospitals that had ATRA available based on whether they were a leukemia treatment center versus a referring center $(40 / 69$ [58\%] vs 7/49 [14\%], respectively; $P=.000002)$. Alarmingly, even for hospitals stating that they treated patients with acute leukemia, $42 \%$ did not have ATRA immediately available in stock for patients.

The survey identified 3 common barriers to the availability of ATRA in hospitals: (1) the drug had not been recently requested by a physician and therefore was not available, (2) the inpatient pharmacist was not familiar with the drug, and (3) the hospital relied on associated hospitals or cancer centers to provide the drug to the patient.

\section{Discussion}

APL is an aggressive subtype of AML characterized by a distinct morphology, cytogenetic chromosomal translocation, clinical presentation, and therapeutic recommendation. ${ }^{1,5,6}$ Although APL is known to be the most curable form of acute leukemia, a high proportion of patients presents with or are at high risk of hemorrhagic and thrombotic complications. As a result, a high early death rate can occur. ${ }^{3}$ The first 24 hours are crucial in diagnosing and instituting treatment with ATRA to reverse DIC, a life-threatening coagulopathy. ATRA can significantly mitigate DIC, and immediate initiation of ATRA therapy and supportive measures aimed at counteracting disease-associated coagulopathy improve early survival. ${ }^{7}$ Delaying ATRA administration seems to contribute to increased hemorrhagic complications and an early death rate. ${ }^{4,7}$ In one report, the incidence of early death in 204 patients with APL was $11 \%$, with most deaths resulting from hemorrhage $(61 \%){ }^{7}$ High-risk patients with APL (defined as WBC count $>10,000 / \mu \mathrm{L}$ ) who received ATRA had a lower overall early death rate. ${ }^{7}$ When ATRA was administered within 24 hours of suspicion of APL, the rate of early death due to hemorrhage was $33 \%$. However, when ATRA was administered $>24$ hours after the initial 
suspicion of the diagnosis, the death rate due to hemorrhage increased to $>70 \%{ }^{7}$

Although national guidelines support the rapid introduction of ATRA as soon as there is a morphologic consideration for APL, patients who are transferred to our center for treatment of APL often experience a delay in initiation of ATRA while awaiting transfer to our center, even when urgent initiation is recommended before transfer. To further investigate the underpinnings of this delay, we randomly surveyed 120 medical centers from 6 different geographic regions in the United States. Our survey was completed by $98 \%(118 / 120)$ of the contacted hospitals, reducing the chance that selection bias or sampling error significantly affected our results. We found that most surveyed hospitals caring for these patients could not rapidly institute therapy because of a lack of availability of the medication. In addition, only $14 \%(7 / 49)$ of surveyed hospitals that typically referred patients with acute leukemia to tertiary care centers could provide ATRA as a bridge before their transfer.

Much has been written regarding the early 30-day mortality seen in patients with APL. However, we cannot specifically comment on whether these findings impact the rates of mortality for patients with APL treated in hospitals without immediate access to ATRA when compared with those with the medication on formulary. Although we determined 3 main reasons for the lack of availability, the underlying cause may simply be the relatively rare incidence of APL (2.7\%) combined with the cost to stock ATRA (average wholesale price of $\$ 2,988.16$ for 100 tablets of $10 \mathrm{mg}$ each), possibly leading to a perception that there is no significant benefit to having ATRA on formulary. ${ }^{8} \mathrm{~A}$ review of the data also illustrates another possible consequence of the current FDA label for ATRA. Tretinoin is currently only FDA-approved for patients with APL who are refractory to or who have relapsed from anthracycline chemotherapy or for whom anthracycline-based chemotherapy is contraindicated. It is not FDA-approved for frontline use or for use in consolidation or maintenance settings. The current FDA approved indications run counter to the NCCN Guidelines, in which ATRA is a part of all frontline therapeutic recommendations for low-/intermediate-risk and high-risk disease. ${ }^{6}$ Thus, manufacturers cannot promote the off-label use of ATRA and cannot provide assistance programs for its off-label use despite its universal acceptance as an invaluable asset for the care and management of patients with APL.
This study represents a verbal reporting of accessibility to ATRA based on a telephone survey. We attempted to reach individuals knowledgeable about the formulary at each institution, and therefore focused our discussions primarily with an inpatient pharmacist at each institution. However, as noted in the "Results" section, one reason for the lack of availability of ATRA was a lack of familiarity with the product, which could lead to inaccuracies originating from the reporting practitioner about whether the medication was truly available at the hospitals. Although most institutions require 24 to 48 hours to make medical requests for and to acquire nonformulary medications, we did not specifically inquire as to the time frame for procuring ATRA at centers reporting the medication as nonformulary. Our results are similar to findings from a survey of the hospitals in Michigan and Louisiana that reportedly treat patients with APL, which found that $26 \%$ (6/23) of these hospitals had ATRA on formulary and that the most common reason for not having ATRA on formulary was that the pharmacist was unfamiliar with the drug. ${ }^{9}$

\section{Conclusions}

After surveying 120 randomly selected hospitals, our study suggests that ATRA may not be available at many hospitals across the United States and that this may be a substantially larger issue at referring hospitals. Hospitals in the central and southeastern United States were the least likely to have ATRA available. Our findings should spur an investigation into the impact of early access to ATRA on morbidity and mortality in APL. Furthermore, a call from hematologists nationwide to their formulary committees is warranted to ensure that this lifesaving medication is available to patients in a timely manner.

Submitted August 26, 2020; final revision received January 4, 2021; accepted for publication January 23, 2021

Published online August 17, 2021.

Author contributions: Data collection and analysis: Bixby. Manuscript analysis, writing, and editing: All authors.

Disclosures: The authors have disclosed that they have not received any financial consideration from any person or organization to support the preparation, analysis, results, or discussion of this article.

Correspondence: Dale Bixby, MD, PhD, Division of Hematology and Medical Oncology, Department of Internal Medicine, Michigan Medicine and University of Michigan Medical School, F4811A University Hospital South, 1500 East Medical Center Drive, Ann Arbor, MI 48109

Email: dbixby@umich.edu

\section{References}

1. Tallman MS, Altman JK. How I treat acute promyelocytic leukemia. Blood 2009;114:5126-5135. https://doi.org/10.1182/blood-2009-07-216457

2. Dores GM, Devesa SS, Curtis RE, et al. Acute leukemia incidence and patient survival among children and adults in the United States, 2001-2007. Blood 2012;119:34-43. https://doi.org/10.1182/blood-2011-04-347872
3. Park JH, Qiao B, Panageas KS, et al. Early death rate in acute promyelocytic leukemia remains high despite all-trans retinoic acid. Blood 2011;118:12481254. https://doi.org/10.1182/blood-2011-04-346437

4. Lehmann S, Ravn A, Carlsson L, et al. Continuing high early death rate in acute promyelocytic leukemia: a population-based report 
from the Swedish Adult Acute Leukemia Registry. Leukemia 2011;25: 1128-1134. https://doi.org/10.1038/leu.2011.78

5. Sanz MA, Fenaux P, Tallman MS, et al. Management of acute promyelocytic leukemia: updated recommendations from an expert panel of the European LeukemiaNet. Blood 2019;133:1630-1643. https://doi.org/10.1182/blood2019-01-894980

6. Tallman MS, Wang ES, Altman JK, et al. NCCN Clinical Practice Guidelines in Oncology: Acute Myeloid Leukemia. Version 3.2021. Accessed July 19, 2021. To view the most recent version, visit NCCN.org
7. Altman JK, Rademaker A, Cull E, et al. Administration of ATRA to newly diagnosed patients with acute promyelocytic leukemia is delayed contributing to early hemorrhagic death. Leuk Res 2013;37:1004-1009. https://doi. org/10.1016/j.leukres.2013.05.007

8. IBM Micromedex. Red Book drug references. Accessed July 19, 2021. Available at: http://www.micromedexsolutions.com

9. Bolds SL, Hassan SMK, Caprara CR, et al. Availability of all-trans retinoic acid and support systems for management of acute promyelocytic leukemia in Michigan and Louisiana, USA. Hematol Rep 2019;11:7896. https://doi.org/ $10.4081 / \mathrm{hr} .2019 .7896$ 